\title{
Mechanism of the Diastolic Dysfunction Induced by Glycolytic Inhibition Does Adenosine Triphosphate Derived from Glycolysis Play a Favored Role in Cellular $\mathrm{Ca}^{2+} \mathrm{Homeostasis}$ in Ferret Myocardium?
}

\author{
Hideo Kusuoka and Eduardo Marban \\ Division of Cardiology, Department of Medicine, The Johns Hopkins University School of Medicine, Baltimore, Maryland 21205
}

\begin{abstract}
Several lines of evidence indicate that glycolysis is especially important for normal diastolic relaxation and for the maintenance of cellular ion homeostasis in myocardium. To elucidate whether the glycolytic flux of ATP contributes to diastolic tone and to the regulation of intracellular $\mathrm{Ca}^{2+}$, myocardial content of sugar phosphates ([SP]) and intracellular $\mathrm{Ca}^{2+}$ concentration $\left(\left[\mathrm{Ca}^{2+}\right]_{i}\right)$ were measured in isolated, perfused ferret hearts using nuclear magnetic resonance. Glucose and acetate were used as substrates for glycolysis and oxidative phosphorylation, respectively. Glycogen was effectively depleted after 15min perfusion with glucagon ( $2 \mathrm{mg} / \mathrm{liter}$ ), as verified by the lack of rise in [SP] during exposure to iodoacetate $(100 \mu \mathrm{M})$ in substrate-free perfusate. Despite the fact that glycolytic flux had been blocked both by iodoacetate and by absence of substrate, end-diastolic left ventricular pressure (EDP) remained unchanged $(P>0.15, n=6)$. The subsequent addition of glucose to the perfusate led to SP accumulation and a marked rise in EDP, with a significant correlation between EDP and [SP] $(r$ $=0.86 \pm 0.04, P<0.01, n=6)$. A similar correlation was observed when glucose in the perfusate was replaced by 2-deoxyglucose $(r=0.78 \pm 0.09, P<0.01, n=3)$. Fluorine nuclear magnetic resonance measurements of $\left[\mathrm{Ca}^{2+}\right]_{i}$ verified that EDP faithfully reports changes in diastolic $\left[\mathrm{Ca}^{2+}\right]_{i}$ under the present experimental conditions. Thus, intracellular $\mathrm{Ca}^{2+}$ overload is caused by the accumulation of SP rather than by the inhibition of glycolysis per se. Glycolysis may appear to be important because its by-products are deleterious, and not necessarily because glycolytically derived ATP plays a favored role in ion homeostasis. (J. Clin. Invest. 1994. 93:1216-1223.) Key words: glycolysis $\bullet$ intracellular calcium concentration • nuclear magnetic resonance spectroscopy $\bullet$ sugar phosphates
\end{abstract}

\section{Introduction}

Although evaluation of cardiac contractility has traditionally focused on systole, diastolic properties figure just as promi-

A part of this study was presented at the 1992 Scientific Sessions of the American Heart Association and reported in abstract form (1992. Circulation. 86:I-748).

Dr. Kusuoka's current address is Biomedical Research Center, Osaka University Medical School, Suita, Osaka, 565 Japan.

Address correspondence to Dr. Eduardo Marban, Department of Medicine, 844 Ross Building, The Johns Hopkins University School of Medicine, 720 N. Rutland Avenue, Baltimore, MD 21205.

Received for publication 17 May 1993 and in revised form $25 \mathrm{Au}$ gust 1993.

J. Clin. Invest.

(c) The American Society for Clinical Investigation, Inc.

$0021-9738 / 94 / 03 / 1216 / 08 \$ 2.00$

Volume 93, March 1994, 1216-1223 nently in modern conceptualizations of cardiac function. The old idea that diastolic dysfunction, when present, could be traced to arcane mechanical factors such as pericardial constriction, has yielded to the realization that common biochemical alterations in the myocardium can affect relaxation (1). Changes in intracellular $\mathrm{Ca}^{2+}$ homeostasis have been particularly implicated as potential causes of diastolic dysfunction in acute ischemia and in chronic cardiomyopathy $(2,3)$, but the basis of such changes remains uncertain. Of particular interest is the possibility that changes in energy metabolism might perturb ion homeostasis, since the provision of high-energy phosphates is altered in ischemia and possibly in congestive heart failure (4).

Several lines of evidence indicate that glycolysis may be especially important for the maintenance of cellular ion homeostasis and, by implication, for diastolic relaxation. In vascular smooth muscle, lactate is produced even under aerobic conditions and solely from exogenous glucose $(5,6)$, supporting the idea that glycolysis plays an important role in this tissue. Indeed, glycolysis has been proposed to provide ATP for preferential utilization by the $\mathrm{Ca}^{2+}$ pump (7) and $\mathrm{Na}^{+}-\mathrm{K}^{+} \operatorname{pump}(5,6)$ in plasma membrane. Similar compartmentation of energy source has also been reported in the myocardium (8), where inhibition of oxidative phosphorylation mainly suppresses systolic force generation. In contrast, glycolytic inhibition induces a marked loss of $\mathrm{K}^{+}$from the cells and a pronounced rise in diastolic tension ("contracture"), suggesting that energy from glycolysis is preferentially used to support ion transport in myocardium. Recently, Weiss and Lamp $(9,10)$ have provided direct evidence that glycolytically derived ATP can preferentially inhibit ATP-sensitive $\mathrm{K}^{+}$channels in the sarcolemma of cardiac myocytes.

Abnormalities in intracellular $\mathrm{Ca}^{2+}$ handling during ischemia and in the early phase of reperfusion (11-14) also appear to be importantly influenced by anaerobic metabolism. Glycolysis not only helps maintain a low diastolic tone during ischemia $(15,16)$, but also fosters recovery of diastolic function upon reperfusion (17-19). Such beneficial effects of glycolysis have been attributed to a preferential ability of glycolytically derived ATP to restore calcium homeostasis (20). Other lines of evidence have also hinted at linkages between glycolytic inhibition and abnormal $\mathrm{Ca}^{2+}$ homeostasis (21-23).

Nevertheless, the precise role of glycolysis in diastolic dysfunction or abnormal handling of intracellular $\mathrm{Ca}^{2+}$ is not yet clear. We undertook this study to determine whether the glycolytic flux of ATP contributes to diastolic tone and to the regulation of intracellular $\mathrm{Ca}^{2+}$. We find that glycolytic inhibition causes intracellular calcium overload and an increase in diastolic stiffness, but only when glycolytic intermediates are allowed to accumulate in the myocardium. Thus, phosphorylated metabolites, not deficiency of glycolytically derived ATP, may account for the deleterious consequences of glycolytic inhibitors. A preliminary report has appeared (24). 


\section{Methods}

The method for the perfusion of isolated hearts has been described in detail (25). Briefly, hearts were excised from male ferrets (10-14 wk of age ) that had been anesthetized with sodium pentobarbital and heparinized, and Langendorff-perfused at $30^{\circ} \mathrm{C}$ with a modified Tyrode solution. The following composition (in $\mathrm{mM}$ ) is for the standard perfusate: $\mathrm{NaCl} 108, \mathrm{KCl} 5, \mathrm{MgCl}_{2} 1$, Hepes $5, \mathrm{CaCl}_{2} 2$, glucose 10 , and sodium acetate 20 . The $\mathrm{pH}$ was adjusted to 7.4 by titration with $\mathrm{NaOH}$. The perfusate was bubbled continuously with $100 \% \mathrm{O}_{2}$. A thin latex balloon tied to the end of a polyethylene tube was inserted into the left ventricle through the mitral valve and connected to a pressure transducer (Statham P23ID, Gould, Inc., Oxford, CA). The balloon was filled either with $15 \mathrm{mM}$ magnesium trimetaphosphate for ${ }^{31} \mathrm{P}$-nuclear magnetic resonance (NMR) ${ }^{1}$ measurements or with $1 \mathrm{mM}$ 6-fluorotryptophan (6F-Trp) as a standard for the calcium measurements by ${ }^{19} \mathrm{~F}-\mathrm{NMR}$ spectroscopy. The balloon volume was set to achieve an initial end-diastolic pressure (EDP) of $5-12 \mathrm{mmHg}$, then kept isovolumic throughout the experiment. Left ventricular pressure was recorded with a direct-writing recorder. After 10-20 min of stabilization, coronary flow rate, controlled by a peristaltic pump, was adjusted such that perfusion pressure equalled $80-90 \mathrm{mmHg}$. Once adjusted, the flow rate was kept constant throughout the experiment. The heart rate was maintained at $2-3 \mathrm{~Hz}$ by right ventricular pacing with an agar wick soaked in saturated $\mathrm{KCl}$, encased in polyethylene tubing, and connected to a stimulator. To obtain the NMR signals, the preparation was lowered into a 25-mm diam NMR tube and placed into the wide-bore superconducting magnet of a spectrometer ( 8.1 Tesla; AM-360, Bruker Instruments, Billerica, MA). Research protocols were approved by the Animal Care and Use Committee of the institution and animal experiments were performed in accordance with the guiding principles of the American Physiological Society.

Measurement of myocardial contents of energy-related phosphorus compounds. Intramyocardial contents of sugar phosphates (SP), inorganic phosphate $\left(\mathrm{P}_{\mathrm{i}}\right)$, phosphocreatine $(\mathrm{PCr})$, and $\mathrm{ATP}$, as well as intracellular $\mathrm{pH}\left(\mathrm{pH}_{\mathrm{i}}\right)$, were measured using ${ }^{31} \mathrm{P}$-NMR spectroscopy. As described previously (25), minimally saturated ${ }^{31} \mathrm{P}-\mathrm{NMR}$ spectra were obtained at a spectral width of $6 \mathrm{kHz}$ using $45^{\circ}$ pulses delivered every $2 \mathrm{~s}$. The spectra were accumulated for $5 \mathrm{~min}$ and processed as described (25). The amounts of SP, $\mathrm{P}_{\mathrm{i}}, \mathrm{PCr}$, and ATP in the myocardium were obtained by planimetry of the areas under individual peaks. The tissue contents of SP, $\mathrm{P}_{\mathrm{i}}, \mathrm{PCr}$, and ATP were normalized using the signal of trimetaphosphate in the left ventricular balloon, then divided by the wet weight of each heart. When the standard for ${ }^{31} \mathrm{P}-\mathrm{NMR}$ measurements was not available, the tissue contents of $S P, P_{i}, P C r$, and ATP were expressed as percent of the area under the ATP peak during the initial control period in each individual experiment (26). Intracellular $\mathrm{pH}$ was determined from the chemical shift of the $P_{i}$ peak measured relative to the resonance of $\mathrm{PCr}$ (25).

Measurement of intracellular free calcium concentration at enddiastole. As previously described $(27,28)$, the $\mathrm{Ca}^{2+}$ indicator $5 \mathrm{~F}$ BAPTA, the 5,5'-difluoro form of 1,2-bis(2-aminophenoxy) ethane$N, N, N^{\prime}, N^{\prime}$-tetraacetic acid (29), was loaded into cells by perfusion with its tetra-acetoxymethyl ester derivative (5F-BAPTA-AM). In this study, we added $12.5 \mu \mathrm{M}$ 5F-BAPTA-AM (Molecular Probes, Inc., Eugene, OR) to the perfusate for 15-17 $\mathrm{min}$ to achieve a favorable signal-to-noise ratio in a ${ }^{19} \mathrm{~F}$-NMR spectrum. The perfusate was then switched to a modified Tyrode solution with extracellular Ca concentration $\left([\mathrm{Ca}]_{\mathrm{o}}\right)$ equal to $8 \mathrm{mM}$ to antagonize partially the negative inotropic effect of the calcium buffering induced by 5F-BAPTA (28),

1. Abbreviations used in this paper: 5F-BAPTA, 5,5'-difluoro 1,2bis(2-aminophenoxy)ethane- $N, N, N, N^{\prime}$-tetraacetic acid; DOG, 2-deoxyglucose; EDP, end-diastolic left ventricular pressure; 6F-Trp, 6fluorotryptophan; IAA, iodoacetate; NMR, nuclear magnetic resonance; $\mathrm{PCr}$, phosphocreatine; $\mathrm{pH}_{\mathrm{i}}$, intracellular $\mathrm{pH} ; \mathrm{P}_{\mathrm{i}}$, inorganic phosphate; $\mathrm{SP}$, sugar phosphates. and supplemented with $1 \mathrm{mM}$ probenecid (Sigma Chemical Co., St. Louis, MO) to minimize the time-dependent extrusion of 5F-BAPTA from myocardial cells (28).

The methods for measuring intracellular free calcium concentration $\left(\left[\mathrm{Ca}^{2+}\right]_{\mathrm{i}}\right)$ with temporal resolution during the cardiac cycle have been described in whole hearts using gated ${ }^{19} \mathrm{~F}$ NMR $(11,28)$. In this study, we concentrated on end-diastolic $\left[\mathrm{Ca}^{2+}\right]_{i}$; thus, free induction decays were acquired $20 \mathrm{~ms}$ before electrical stimulation to the heart using a gating technique. The free induction decays from the hearts were accumulated with $1 \mathrm{k}$ data points at a spectral width of about 5 $\mathrm{kHz}$ using $70^{\circ}$ pulses. All chemical shifts were referenced with respect to the signal of $6 \mathrm{~F}-\mathrm{Trp}$, which was assigned a value of 0 parts per million (ppm).

Intracellular free calcium concentration, $\left[\mathrm{Ca}^{2+}\right]_{\mathrm{i}}$, is calculated according to the equation $\left[\mathrm{Ca}^{2+}\right]_{\mathrm{i}}=K_{\mathrm{d}}([B] /[F])$, where $[B]$ is the area under the Ca-5F-BAPTA peak near $8 \mathrm{ppm}$, and $[F]$ is the area under the Ca-free 5F-BAPTA peak near 2 ppm (see Fig. 8 [27]). We have used a dissociation constant for Ca-5F-BAPTA $\left(K_{d}\right)$ of $285 \mathrm{nM}$ to calibrate our signals (10).

Depletion of intramyocardial glycogen. Vander Heide et al. (30) have demonstrated that tissue levels of glycogen can be significantly reduced even by brief ( $3 \mathrm{~min}$ ) exposure to glucagon under substratefree conditions. We confirmed the efficacy of depletion of myocardial glycogen by simple substrate withdrawal (Fig. $1, A-C$ ) and by substrate withdrawal during exposure to glucagon (Fig. $1, D-G$ ). The P-NMR spectra shown in $A-C$ were obtained from one heart in which prolonged substrate-free perfusion without glucagon was used in a futile attempt to deplete glycogen. The spectrum in $B$, acquired after $45 \mathrm{~min}$ of perfusion without substrates, is almost identical to that obtained during the initial perfusion with normal solution $(A)$. After the $45 \mathrm{~min}$ of substrate-free perfusion, the heart was exposed to iodoacetate (IAA; $150 \mu \mathrm{M}$ ) for $15 \mathrm{~min}$ to block glycolysis irreversibly at the level of glyceraldehyde 3-phosphate dehydrogenase (31); during this period, acetate was also present as a substrate for oxidative phosphorylation. The spectrum in $C$ was taken when the perfusate was switched back to one with no substrates to stimulate glycogenolysis and glycolysis; the peak at $\approx 6.5 \mathrm{ppm}$ clearly increased. During glycolytic inhibition, this peak has been shown to consist of SP (32). Thus, the results indicate incomplete depletion of glycogen, because glycogen would have been the only source for the generation of SP in the absence of exogenous substrates.

The P-NMR spectra shown in $D-G$ were obtained from another heart in which glucagon was used to aid in the depletion of glycogen. The spectrum in $E$, obtained after washout of glucagon, is identical to that in control $(D)$. Furthermore, the spectrum in $F$, taken after exposure to IAA and no substrates, shows little change, confirming that glycogen had indeed been depleted. Nevertheless, when glucose was readded to the perfusate, sugar phosphates clearly accumulated $(G)$ as a consequence of the glycolytic block. Thus, 15-min perfusion with glucagon and no substrates effectively depletes intramyocardial glycogen.

Experimental design. After initial stabilization in normal perfusate, P-NMR spectra were taken for control, and then hearts were perfused with a solution containing glucagon $(2 \mathrm{mg} / \mathrm{liter})$ and no substrates to deplete glycogen in myocardium. Fifteen minutes later, hearts were switched to glucagon-free medium, and perfused for an additional 15 min without substrates. After the depletion of glycogen, the substrates in the perfusate were changed by eliminating or adding either glucose or acetate in different combinations, and P-NMR or F-NMR measurements were performed while monitoring left ventricular pressure; the combination of substrates was randomized to avoid systematic or cumulative time-dependent changes. Glycolysis was blocked either by exposure to IAA $(150 \mu \mathrm{M})$ for 15 min or by substitution of glucose with 2-deoxyglucose (DOG; $10 \mathrm{mM}$ ) (33). When utilized, the $\mathrm{Ca}^{2+}$ indicator 5F-BAPTA was loaded into hearts after the completion of glycogen depletion; only acetate was used as a substrate during the 5F-BAPTA loading period.

Statistical analysis. Data are presented as mean \pm SEM. Statistical analysis was performed using correlation analysis, ANOVA with the 

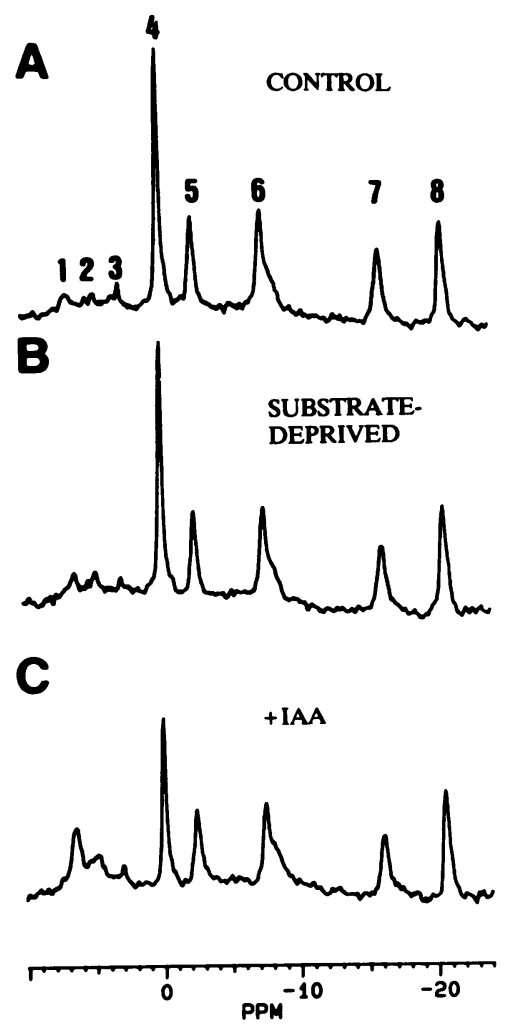

PPM

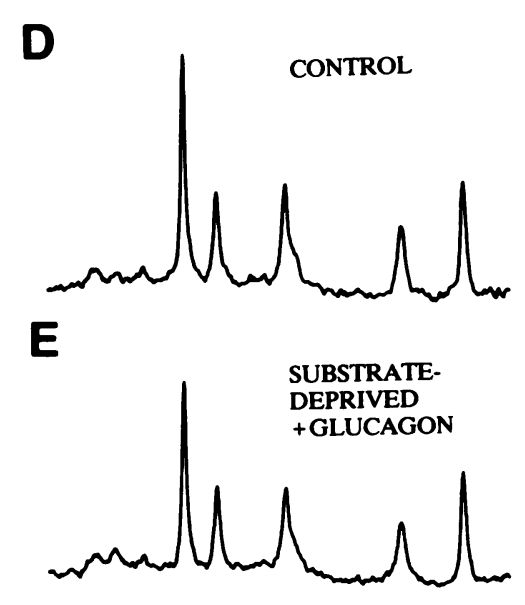

$\boldsymbol{F}$

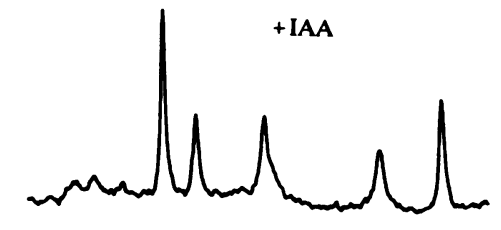

G

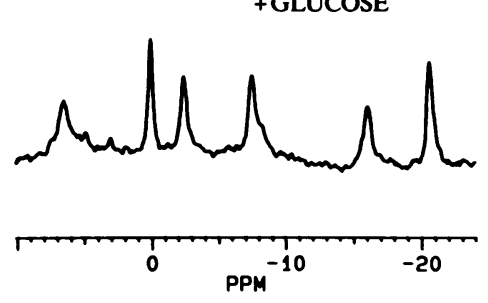

Figure 1. Effect of the blockage of glycolysis in hearts with or without the depletion of glycogen. $(A-C)$ P-NMR spectra obtained from a heart in which glycogen was depleted incompletely by perfusion for $\mathbf{4 5}$ min with no substrates. Spectrum $B$ obtained after $45-\mathrm{min}$ perfusion was almost the same as that obtained during control $(A)$. Spectrum $C$ obtained after 15 -min exposure to IAA ( $150 \mu \mathrm{M})$ and switching to substrate-free perfusate shows the accumulation of SP, indicating incomplete depletion of glycogen. ( $D-G)$ P-NMR spectra obtained from a heart where glycogen was completely depleted. Spectrum $E$, obtained after 15 -min perfusion with glucagon ( $2 \mathrm{mg} /$ liter) and no substrate, was the same as that during control $(D)$. Furthermore, spectrum $F$ obtained after exposure to IAA and no substrate showed little change, indicating complete depletion of glycogen. When glucose was added as substrate, SP accumulated as shown in $G$. The main identified peaks in $A$ are $1, \mathrm{SP} ; 2, \mathrm{P}_{\mathrm{i}} ; 3$, phosphodiester; 4, PCr; 5-7, ATP; 8, trimetaphosphate as standard.
Scheffé test, and regression analysis as appropriate (34). Probability of null hypothesis of $<5 \%(P<0.05)$ was considered significant.

\section{Results}

Effects of glycolytic inhibition on function and phosphorus compounds. First, we tried to elucidate whether the glycolytic flux of ATP per se, or other factors contribute to the maintenance of diastolic tone. Fig. 2 shows results from a typical experiment in which we first depleted glycogen, then blocked glycolysis by exposure to IAA. The top panel indicates the times of exposure to glucagon or IAA, and the changes in substrate composition. Below are plotted the time courses of left ventricular function and intramyocardial phosphorus compounds. After the depletion of glycogen and before glycolytic blockade (i.e., 25-85 min), the peak left ventricular pressure and the diastolic pressure (DP) were well maintained, until the 75-85-min interval when the heart was perfused with neither glucose nor acetate. At that time, $\left[P_{i}\right]$ increased, with concomitant decreases of [PCr] and of systolic pressure. The contractile failure observed here is attributable to the increase of $\left[P_{i}\right](35)$. In contrast, EDP did not vary significantly with the alterations in substrate composition.

During the exposure to IAA ( $85-100 \mathrm{~min}$ ) in the absence of substrates, there was only a small increase in sugar phosphates, indicating that glycogen had been nearly completely depleted.
Ventricular function recovered substantially when acetate was reintroduced to the perfusate $(100 \mathrm{~min})$; in particular, EDP remained low throughout. The latter finding is notable since glycolytic flux of ATP was prevented not only by substrate depletion but also by the IAA-induced inactivation of glyceraldehyde 3-phosphate dehydrogenase. The observation that diastolic function remained normal, which was confirmed in 6 hearts, clearly dissociates ventricular diastolic tone from an obligatory dependence on ATP derived from glycolysis.

Subsequent reintroduction of glucose (at $110 \mathrm{~min}$ ) led to a brisk increase in concentration of sugar phosphates. Interestingly, DP increased as SP accumulated in the cytosol. Diastolic pressure recovered partially when glucose was once again removed from the medium (130-155 $\mathrm{min})$, in parallel with a decrease of [SP]. However, when glucose was reintroduced, both [SP] and EDP increased dramatically and progressively (155-180 $\mathrm{min}$ ).

Fig. 2 points out two important concepts. First, glycolytically derived ATP is not critical for intracellular $\mathrm{Ca}^{2+}$ regulation. (EDP was used as a bioassay of diastolic $\mathrm{Ca}^{2+}$ levels; the validity of this bioassay is confirmed later.) Second, SP accumulation itself may be deleterious for $\mathrm{Ca}^{2+}$ homeostasis. When the heart was perfused only with glucose after the inhibition of glycolysis by IAA (120-125, 155-165, and 175-185 min), SP did accumulate as expected, and EDP increased. These results 

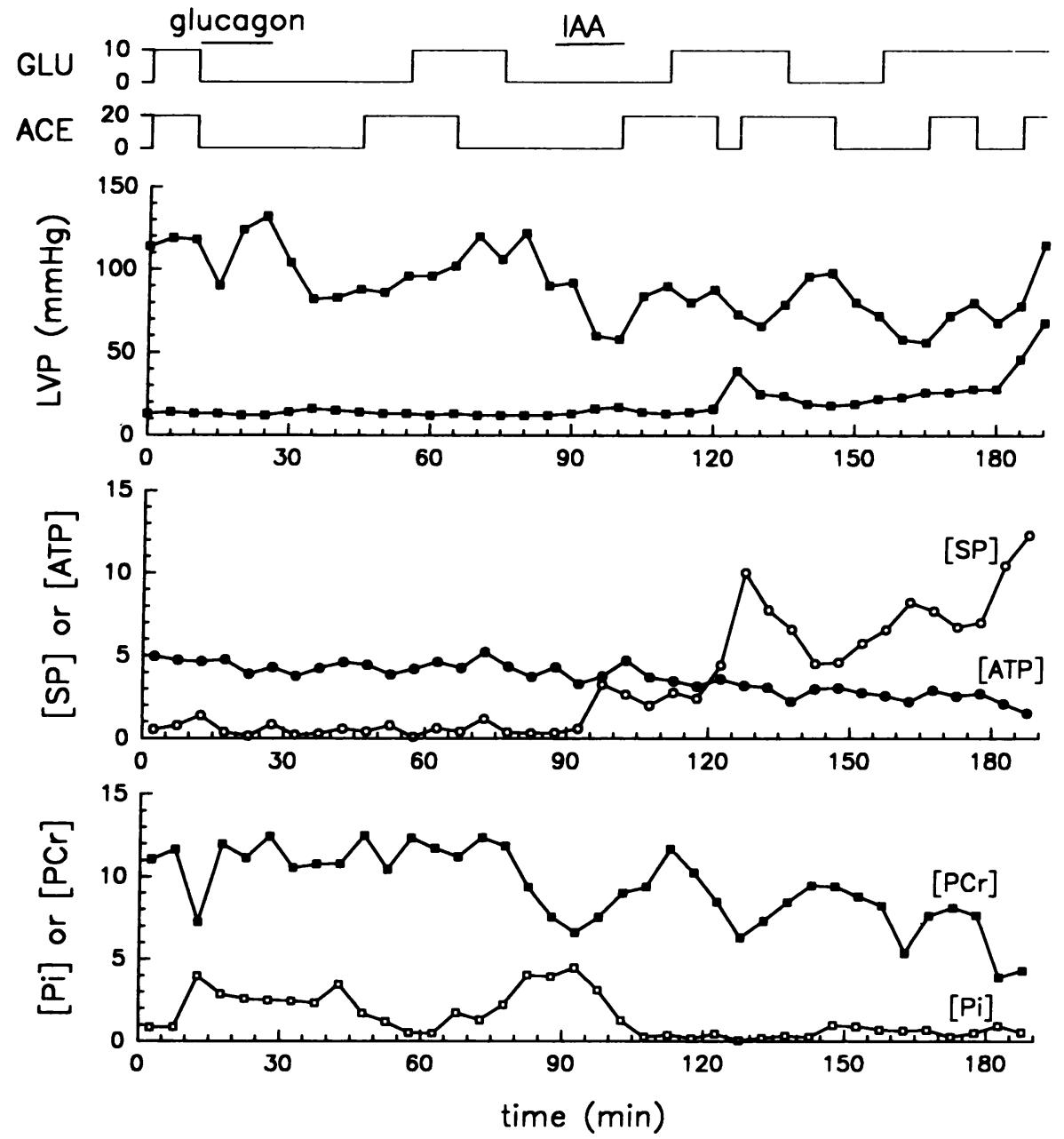

Figure 2. Changes in left ventricular pressure $(L V P)$ and intramyocardial phosphorus compounds induced by different pairs of substrates. The top panel indicates a pair of substrates in perfusate; glucose $(G L U)$ and acetate $(A C E)$. Glucagon $(2 \mathrm{mg} /$ liter $)$ and IAA $(150 \mu \mathrm{M})$ were added as indicated. The second panel shows peak and EDPs of left ventricle. The lower panels show intramyocardial contents of sugar phosphates (SP; ○), ATP $(\bullet)$, phosphocreatine $(\mathrm{PCr} ; \bullet)$, and inorganic phosphate $\left(P_{i} ; \square\right)$. The units for SP, ATP, PCr, and $P_{i}$ are $\mu \mathrm{mol} /$ g wet wt.

suggest that accumulation of SP is necessary for EDP to increase; absence of glycolytic flux per se is not sufficient.

Fig. 3 specifically examines the correlation between [SP] and EDP. Time-averaged values of EDP (mean EDP) during each P-NMR acquisition are plotted as a function of the corresponding [SP]. Mean EDP and [SP] were both low before exposure to IAA $(O)$; not surprisingly, there was no correlation between the two at this time $(r=-0.12, P>0.05, n=17)$. In contrast, the increase of diastolic tone occurred hand-in-hand with the increase in SP after blockage of glycolysis $(r=0.76, P$ $<0.01, n=22$ ). This type of analysis, while compelling, examines the correlation of EDP with only one metabolite, while ignoring others which also clearly changed during the experiment. [PCr], [ATP], and $\mathrm{pH}_{\mathrm{i}}$ all correlated with mean EDP, although with somewhat lower coefficients of correlation than [SP] $(r=-0.70$ for [PCr] $P<0.01 ; r=-0.74$ for [ATP], $P$ $<0.01 ; r=-0.49$ for $\left.\mathrm{pH}_{\mathrm{i}}, P<0.05\right)$. Among these other factors, the strongest correlations, those with [PCr] and [ATP], were negative. Such correlations are not unexpected even if SP are of primary importance: after all, the phosphorylation of glycolytic intermediates occurs at the expense of high energy phosphates. The role of these other factors in the diastolic changes will be considered in more detail below.

Effects of different combinations of substrates on EDP and [ $S P$ ]. The changes in EDP and SP shown in Fig. 2 were consistently observed in a total of six experiments. Fig. 4 summarizes the rates of change of $\mathrm{EDP}(d[\mathrm{mEDP}] / d t)$ and $\mathrm{SP}(d[\mathrm{SP}] / d t)$ induced by the different combinations of substrates indicated at the bottom of the figure. Before glycolytic inhibition by IAA (open bars), $d(\mathrm{mEDP}) / d t$ did not depend on the choice of

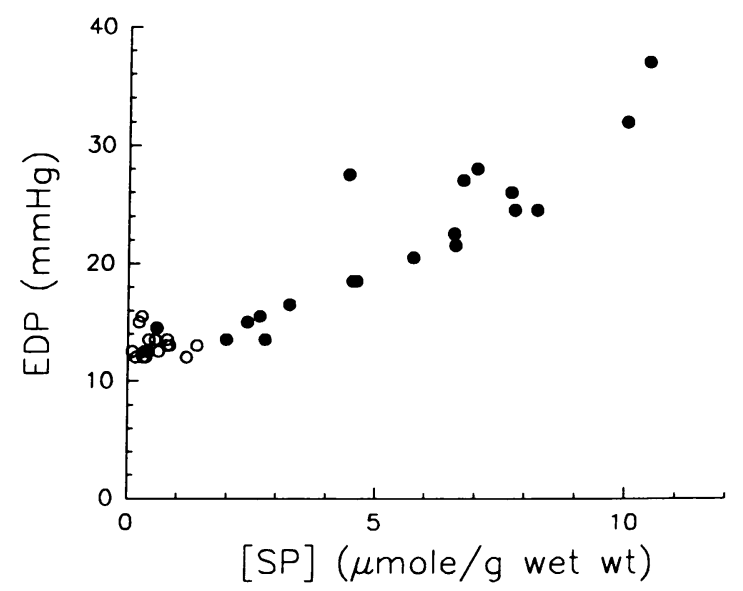

Figure 3. Relationship between intramyocardial amount of SP and EDP. EDP did not correlate with [SP] before exposure to IAA (O; $r=-0.12, P>0.05)$. In contrast, the relation showed significant correlation after blockage of glycolysis $(\bullet ; r=0.76, P<0.01)$. This relation was plotted from the data in Fig. 2. 


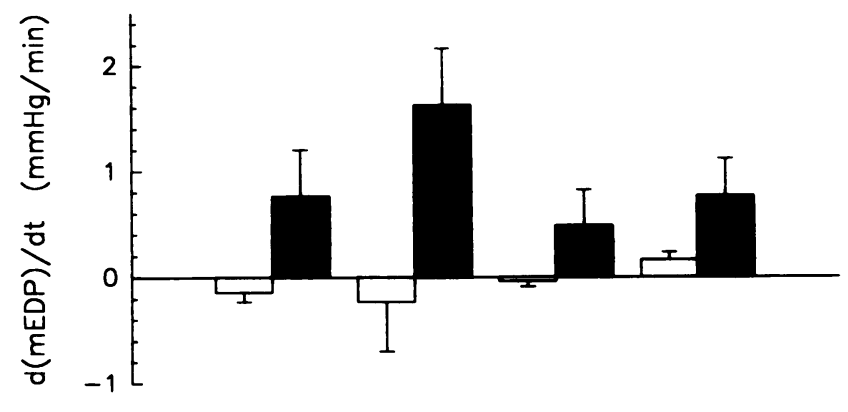

drogenase is sufficient to induce the increase in EDP coupled with SP accumulation.

Contribution of other phosphorus compounds to diastolic dysfunction. To elucidate whether or not phosphorus compounds other than SP contribute to the changes in diastolic tone, the correlation coefficients between EDP and intramyocardial contents of phosphorus compounds were examined (Fig. 6 A). None of $\mathrm{SP}, \mathrm{P}_{\mathrm{i}}, \mathrm{PCr}, \mathrm{ATP}$, or $\mathrm{pH}_{\mathrm{i}}$ showed a signifcant correlation with EDP before glycolysis was inhibited. After glycolytic blockage using either IAA or DOG, sugar phosphates strongly correlated with EDP in all experiments $(r$ $=0.85 \pm 0.04$ after IAA exposure, $r=0.78 \pm 0.09$ in DOG experiments). In contrast, $\mathrm{P}_{\mathrm{i}}, \mathrm{PCr}$, and $\mathrm{pH}_{\mathrm{i}}$ showed a significant correlation with EDP in some experiments, but not consistently. ATP showed a negative correlation with EDP in all experiments $(r=-0.82 \pm 0.04$ after IAA exposure, $r=-0.61 \pm 0.09$ in DOG experiments), suggesting that ATP might also contribute to determine diastolic tone.

To probe a cause-and-effect relationship, correlations between rates of change are more discriminating than simple correlations between steady-state levels. In order for there to be a significant correlation between two rates of change, both fac-

$$
\begin{array}{lllll}
\text { Glu } & (+) & (+) & (-) & (-) \\
\text { Ace } & (+) & (-) & (+) & (-)
\end{array}
$$

Figure 4. The rates of change of EDP or [SP] during perfusion with different combinations of substrates. The upper panel and the lower panel show the averages of the rates of change of mean $\operatorname{EDP}(m E D P)$ and [SP], respectively. Open bars and closed bars indicate the rates before and after glycolytic inhibition, respectively. Glu, glucose; Ace, acetate.

substrates, nor did SP accumulate. After the blockage of glycolysis by IAA (filled bars), both EDP and sugar phosphates increased markedly and significantly during perfusion with glucose only $(d[\mathrm{mEDP}] / d t: 1.62 \pm 0.54 \mathrm{mmHg} / \mathrm{min}, P<0.01$; $d[\mathrm{SP}] / d t: 0.30 \pm 0.08 \mu \mathrm{mol} / \mathrm{g}$ wet $\mathrm{wt} / \mathrm{min}, P<0.005)$; other combinations of substrates induced no significant changes in EDP or SP. Thus, addition of glucose to the perfusate after glycolytic inhibition led to the accumulation of SP and a concomitant rise in EDP. Taken together, these results and those in Fig. 2 support the idea that accumulation of SP is necessary for EDP to increase; block of glycolytic ATP flux without SP accumulation is not sufficient.

Effects of the site blocked in glycolysis on EDP and [SP]. If SP accumulation is linked to the increase in EDP, then EDP should be similarly affected by phosphorylated sugars that accumulate by means other than inhibition of glyceraldehyde 3-phosphate dehydrogenase. To test this hypothesis, phosphoglucose isomerase was blocked by replacing glucose in the perfusate with DOG. Fig. $5 \mathrm{~A}$ shows the time courses of EDP and [SP] from an experiment in which glycogen had been depleted. When the heart was perfused with DOG only (15-30 and 85$110 \mathrm{~min}$ ), SP accumulated and EDP increased. As illustrated in $B$, EDP and SP were correlated significantly $(r=0.91, P$ $<0.01, n=21$ ), similar to our findings in the experiments that used IAA for glycolytic inhibition. The relation between sugar phosphates and EDP induced by DOG was confirmed in two additional experiments. Thus, these results indicate that block of glycolysis at a step before glyceraldehyde 3-phosphate dehy-

\section{A}
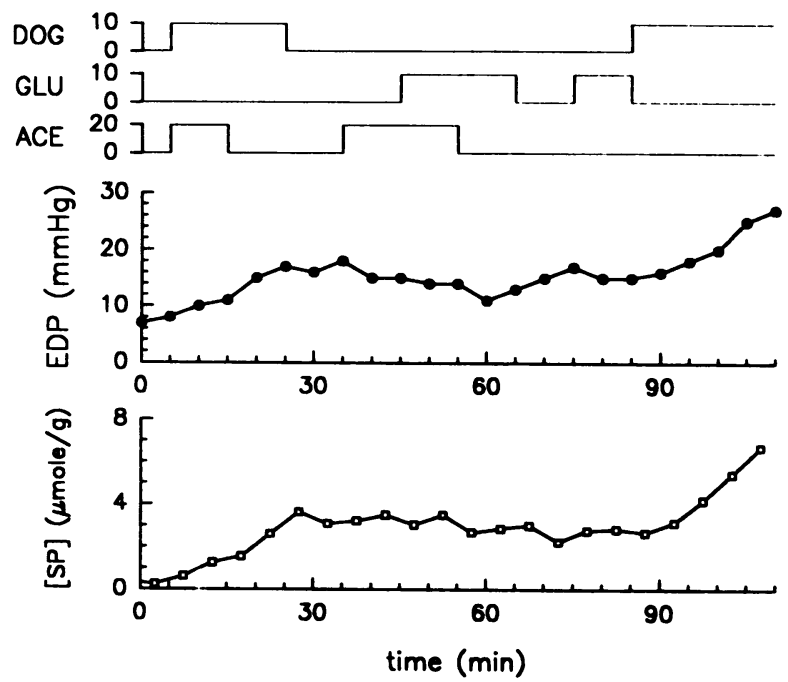

B

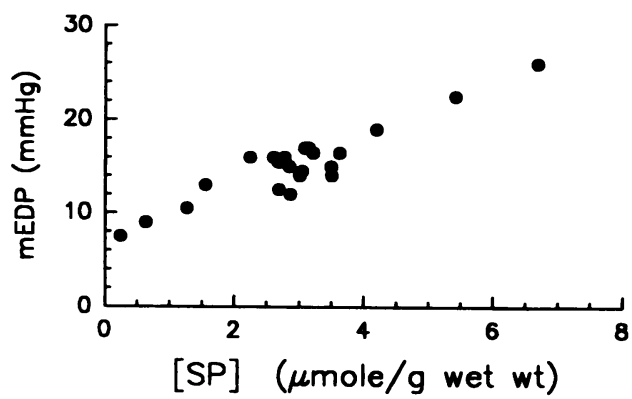

Figure 5. Changes in LVEDP and [SP] in the heart perfused with DOG. $(A)$ The top panel indicates substrates in perfusate after depletion of glycogen; DOG ( $10 \mathrm{mM}$ ) was used to inhibit glycolysis instead of IAA. The middle panel and the lower panel show EDP and [SP], respectively. Note that EDP and [SP] increased only when substrate was DOG only. $(B)$ Relationship between [SP] and EDP in the same heart. The relation showed significant correlation $(r=0.91, P<0.01)$. 

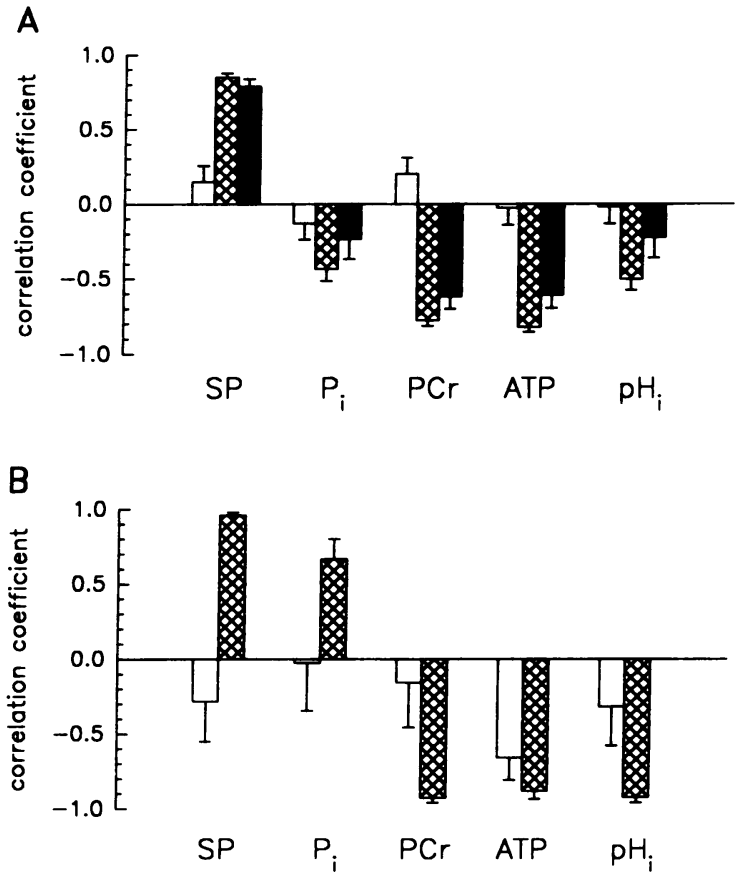

Figure 6. Correlation coefficients between EDP and intramyocardial contents of phosphorus compounds. $(A)$ Correlation coefficients between $\mathrm{mEDP}$ and myocardial concentration of phosphorus compounds in hearts not loaded with 5F-BAPTA. Open bars, hatched bars, and closed bars indicate the average of coefficients before glycolytic inhibition, after inhibition by IAA, and after inhibition by DOG, respectively. $(B)$ Correlation coefficients between mEDP and myocardial concentration of phosphorus compounds in hearts loaded with 5F-BAPTA. Open bars and hatched bars indicate the averages of coefficients before and after inhibition of glycolysis by IAA, respectively.

tors need to have identical temporal and phase relationships. Thus, we examined the correlations among rates of change to determine whether we could establish a stronger link between SP and EDP. The rate of change of EDP $(d[\mathrm{mEDP}] / d t)$ significantly correlated with that of SP $(d[\mathrm{SP}] / d t ; r=0.64 \pm 0.07$ in IAA experiments, $r=0.59 \pm 0.10$ in DOG experiments), whereas there was no correlation with the rate of change of ATP in any of the experiments $(r=-0.31 \pm 0.10$ in IAA experiments, $r=-0.25 \pm 0.16$ in DOG experiments). These results indicate that the myocardial concentration of SP is the major determinant of EDP, whereas the apparent correlation between ATP and EDP is incidental.

Effects of different combinations of substrates on EDP and $[S P]$ in $5 F-B A P T A$ loaded hearts. So far, we have interpreted EDP as a faithful reflection of diastolic intracellular $\mathrm{Ca}^{2+}$ concentration under these experimental conditions. To validate this interpretation, we used the gated F-NMR technique with the $\mathrm{Ca}^{2+}$ indicator 5F-BAPTA. First, we checked whether the correlation between the changes of EDP and that of SP induced by glycolytic inhibition was the same as that in hearts not loaded with 5F-BAPTA. Figure 7A shows the typical changes in EDP and SP in a glycogen-depleted heart loaded with 5FBAPTA. Before glycolytic inhibition (0-40 $\mathrm{min}),[\mathrm{SP}]$ and EDP show little change with or without glucose. After exposure to IAA, SP accumulated when the only substrate was glucose; EDP increased concomitantly, as in hearts not loaded with 5F-BAPTA. [SP] and mEDP after glycolytic inhibition showed a significant correlation (Fig. $7 \mathrm{~B} ; r=0.97, P<0.01, n=12$ ).
The presence of a significant correlation between [SP] and mEDP was confirmed in two other experiments in 5F-BAPTAloaded hearts. Correlation coefficients between mEDP and myocardial concentration of phosphorus compounds in hearts loaded with 5F-BAPTA are depicted in Fig. $6 \mathrm{~B}$. Only the correlation between mEDP and [SP] showed consistent significance after glycolytic inhibition $(r=0.96 \pm 0.17)$, similar to the results in hearts without 5F-BAPTA. These results indicate that the changes in EDP and sugar phosphates in hearts loaded with 5F-BAPTA are identical to those observed in hearts not loaded with 5F-BAPTA.

Intracellular calcium concentration during diastolic dysfunction. Typical spectra of 5F-BAPTA obtained by F-NMR are shown in Fig. 8. The spectrum in $A$ was obtained during diastole from a heart perfused without substrates after the depletion of glycogen (EDP $=12 \mathrm{mmHg}$ ). The peak for free 5F-BAPTA $(F)$ is markedly larger than that for Ca-bound 5FBAPTA $(B)$, indicating that diastolic $\left[\mathrm{Ca}^{2+}\right]_{\mathrm{i}}$ equals $99 \mathrm{nM}$. Panel $B$ shows a spectrum obtained after exposure to IAA, without glucose added to the perfusate (EDP $=33 \mathrm{mmHg}$ ). The peak for the bound form is larger than in the upper panel, and diastolic $\left[\mathrm{Ca}^{2+}\right]_{\mathrm{i}}$ equals $251 \mathrm{nM}$. After addition of glucose, EDP markedly increased to $133 \mathrm{mmHg}$ and the F-NMR spectrum shown in the bottom panel indicates severe $\mathrm{Ca}^{2+}$ overload with $\left[\mathrm{Ca}^{2+}\right]_{\mathrm{i}}$ equal to almost $1 \mu \mathrm{M}$.

The relation between diastolic $\left[\mathrm{Ca}^{2+}\right]_{\mathrm{i}}$ and EDP was deter-

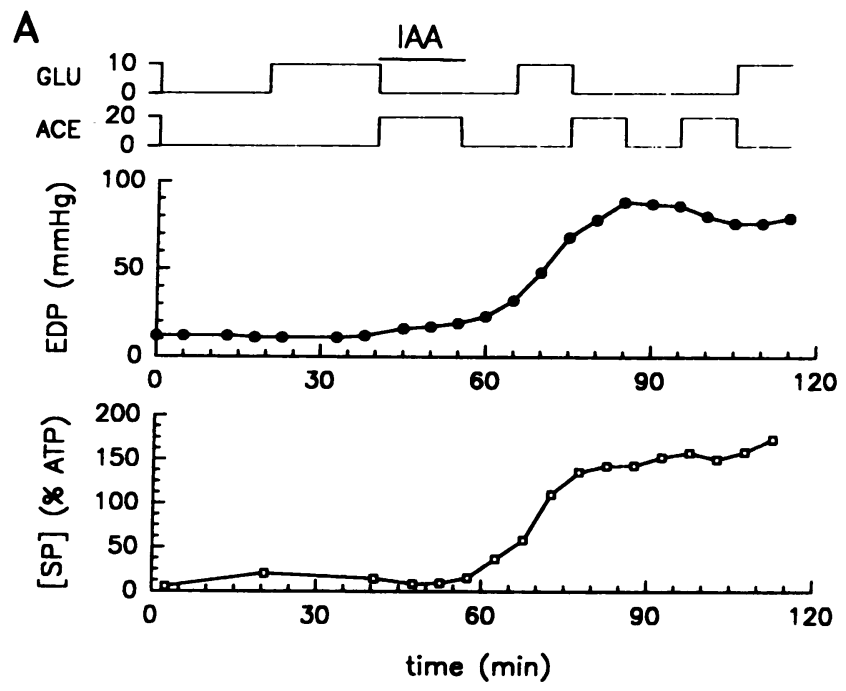

B

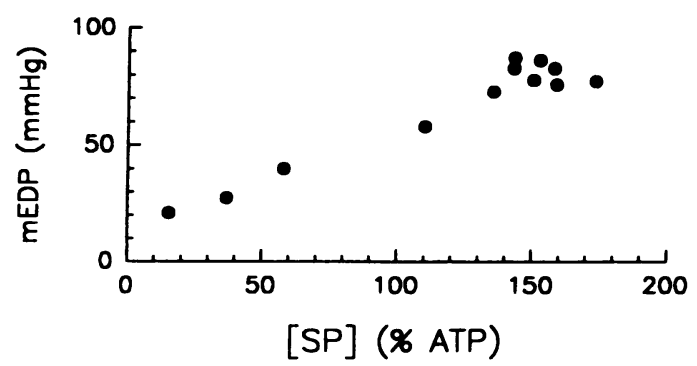

Figure 7. Changes in EDP and [SP] in a heart loaded with 5FBAPTA. $(A)$ The top panel indicates substrates in perfusate after depletion of glycogen. Glycolysis was inhibited by IAA as indicated. The middle panel and the lower panel show EDP and [SP], respectively. GLU, glucose; ACE, acetate. $(B)$ Relationship between [SP] and mEDP after glycolytic inhibition $(r=0.97 ; P<0.05)$. 

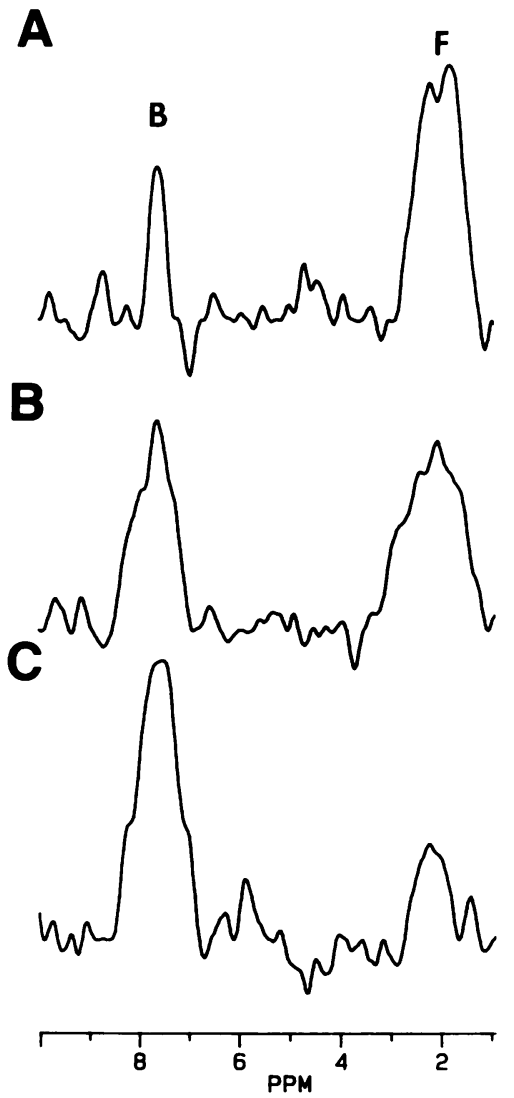

Figure 8. F-NMR spectra obtained during diastolic phase. $(A) \mathrm{F}$ NMR spectrum obtained after the depletion of glycogen, and before the exposure to IAA. Perfusate did not contain glucose. $(B)$ F-NMR spectrum obtained after glycolytic inhibition. Perfusate did not contain glucose. (C) F-NMR spectrum obtained after glycolytic inhibition. Substrate was glucose.

mined in 5 hearts (Fig. 9). Under the present experimental conditions, EDP faithfully reports changes in diastolic $\left[\mathrm{Ca}^{2+}\right]_{i}$ in each individual heart. Thus, these results indicate that the increase in diastolic tone induced by accumulation of SP is attributable to an increase in diastolic $\left[\mathrm{Ca}^{2+}\right]_{i}$. Coupled with the previous results, our data indicate that disruption of intracellular $\mathrm{Ca}^{2+}$ homeostasis is caused by the accumulation of SP, rather than by the interruption of glycolytic ATP production.

\section{Discussion}

The role of ATP in the maintenance of diastolic $\left[\mathrm{Ca}^{2+}\right]_{i}$. Our results indicate that the accumulation of SP directly causes the increase of diastolic $\left[\mathrm{Ca}^{2+}\right]_{i}$, resulting in an increase of diastolic tone. In contrast, glycolytically derived ATP appears to be relatively unimportant. EDP and SP remained unchanged or decreased in parallel under glucose-free conditions, even after the blockage of glycolysis by IAA (for example, 135-145 min in Fig. 2). Furthermore, the rate of change of SP significantly correlated with that of EDP after glycolytic inhibition, but the rate of change of ATP did not show a significant correlation in all experiments.

The deleterious effects of SP accumulation could result from either of two general mechanisms: sugar phosphates themselves might be toxic when they accumulate in the cytosol, such that they interrupt $\mathrm{Ca}^{2+}$ homeostasis; alternativeiy, ATP may be "stolen" to phosphorylate sugars, causing sufficient depletion of ATP to disrupt $\mathrm{Ca}^{2+}$ homeostasis. Although our results do not enable us to distinguish unequivocally between these two possibilities, the ATP steal idea has some difficulties. Presumably the ATP depletion would have to be much

more severe in some local compartment (e.g., a postulated subsarcolemmal space [36]) than it is in the bulk of the myocardium, since bulk ATP depletion of the degree measured in the present study does not, in itself, suffice to compromise ion transport (37). Despite compelling evidence for compartmentation of ATP in some settings, our results argue otherwise. Diastolic tone was well maintained under substrate-free conditions before and after glycolytic inhibition. Thus, ATP generated by oxidative phosphorylation (presumably from endogenous fatty acids [38]) suffices to maintain diastolic ion homeostasis. In fact, ATP appears to be able to go both ways: energy derived from glycolysis can support contraction during the inhibition of oxidative phosphorylation $(39,40)$. If, in the process of accumulation of SP, ATP were consumed locally to such an extent that ion homeostasis was disrupted, this should be a transient phenomenon: in the absence of strict compartmentalization, bulk ATP would soon diffuse to the sites where it is needed. Contrary to this prediction, diastolic tone did not recover even after glucose was removed from the perfusate. EDP stayed high as long as SP remained elevated.

Beneficial effects of glycolytic ATP against metabolic stress. Our results need to be interpreted in light of several lines of evidence suggesting a special role for glycolysis. Glycolytically derived ATP appears to protect against anoxic stress; membrane integrity during hypoperfusion was maintained by perfusion with glucose $(41,42)$. Glucose and insulin preserved diastolic function during underperfusion and subsequent reperfusion (19). The rate of glycolytic flux from glucose correlated well with prevention of ischemic contracture (15); likewise, functional recovery after ischemia could be improved by an increase of glucose oxidation (18). Nevertheless, the mechanism for these beneficial effects of glycolysis has not been clarified. In smooth muscle, glycolysis has been proposed to generate ATP specifically to support the transport of $\mathrm{Na}^{+}, \mathrm{K}^{+}(5,6$, 43 ), and $\mathrm{Ca}^{2+}(7)$ across the plasma membrane. Functional compartmentation of ATP similar to that observed in smooth muscle has also been proposed in myocardium ( 8-10); glycolysis has been argued to preferentially support ion homeostasis, whereas oxidative phosphorylation contributes more to force generation. Results with glycolytic inhibitors have been used to bolster the notion that glycolytically derived ATP maintains intracellular $\mathrm{Ca}^{2+}$ homeostasis and protects against calcium overload during reperfusion $(20,21,23)$. The positive correlation between $\mathrm{Ca}^{2+}$ overload and [SP] during ischemia, and the negative correlation between functional recovery and [SP] in reperfused myocardium (44), support the idea that SP may

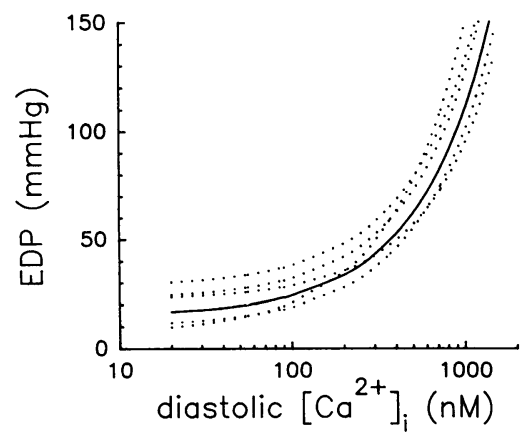

Figure 9. Relation between diastolic $\left[\mathrm{Ca}^{2+}\right]_{\mathrm{i}}$ and EDP. The relation between diastolic $\left[\mathrm{Ca}^{2+}\right]_{\mathrm{i}}$ and mean EDP derived from five hearts is presented. The dotted lines indicate the regression obtained from each individual heart, while the solid line indicates the regression obtained by fitting the pooled

data from all hearts: $\operatorname{EDP}(\mathrm{mmHg})=2237 \cdot \exp (0.04168 \cdot$ $\left.\left[\mathrm{Ca}^{2+}\right]_{\mathrm{i}}(\mathrm{nM})\right)-2222$. 
importantly influence $\mathrm{Ca}^{2+}$ homeostasis in ischemic and/or reperfused myocardium.

We have shown that cardiac function, particularly diastolic function, can proceed unimpaired even when glycolysis is incapable of producing any ATP. Particular caution is merited in the interpretation of previous work using glycolytic inhibitors: glycolysis may have appeared to be important because its byproducts were deleterious, and not necessarily because glycolytically derived ATP plays a favored role. Nevertheless, our results do not exclude the possibility that enhanced glycolytic ATP production has favorable effects, nor do they disprove the notion that there is some degree of functional compartmentation of energy metabolism in heart cells.

\section{Acknowledgments}

The authors thank Dr. Brian O'Rourke for helpful discussions.

This study was supported by RO1 HL-44065 (to EM) from the National Institutes of Health.

\section{References}

1. Grossman, W. 1991. Diastolic dysfunction in congestive heart failure. $N$. Engl. J. Med. 325:1557-1564.

2. Beuckelmann, D. J., M. Naubauer, and E. Erdmann. 1992. Intracellular calcium handling in isolated ventricular myocytes from patients with termina heart failure. Circulation. 85:1046-1055.

3. Gwathmey, J. K., M. T. Slawsky, R. J. Hajjar, G. M. Briggs, and J. P. Morgan. 1990. Role of intracellular calcium handling in force-interval relationships of human ventricular myocardium. J. Clin. Invest. 85:1599-1613.

4. Conway, M. A., J. Allis, R. Ouwerkerk, T. Niioka, B. Rajagopalan, and G. K. Radda. 1991. Detection of low phosphocreatine to ATP ratio in failing hypertrophied human myocardium by ${ }^{31} \mathrm{P}$ magnetic resonance spectroscopy. Lancet. 338:973-976.

5. Lynch, R. M., and R. J. Paul. 1987. Compartmentation of carbohydrate metabolism in vascular smooth muscle. Am. J. Physiol. 252:C328-C334.

6. Campbell, J. D., and R. J. Paul. 1992. The nature of fuel provision for the $\mathrm{Na}^{+}, \mathrm{K}^{+}$-ATPase in porcine vascular smooth muscle. J. Physiol. (Lond.). 447:67-82.

7. Paul, R. J., C. D. Hardin, L. Raeymaekers, F. Wuytack, and R. Casteels. 1989. Preferential support of $\mathrm{Ca}^{2+}$ uptake in smooth muscle plasma membrane vesicles by an endogenous glycolytic cascade. FASEB J. 3:2298-2301

8. Weiss, J., and B. Hiltbrand. 1985. Functional compartmentation of glycolytic versus oxidative metabolism in isolated rabbit heart. J. Clin. Invest. 75:436447.

9. Weiss, J. N., and S. T. Lamp. 1987. Glycolysis preferentially inhibits ATPsensitive $\mathrm{K}^{+}$channels in isolated guinea pig cardiac myocytes. Science (Wash. DC). 238:67-69.

10. Weiss, J. N., and S. T. Lamp. 1989. Cardiac ATP-sensitive $\mathrm{K}^{+}$channels. Evidence for preferential regulation by glycolysis. J. Gen. Physiol. 94:911-935.

11. Marban, E., M. Kitakaze, Y. Koretsune, D. T. Yue, V. P. Chacko, and M. M. Pike. 1990. Quantification of $\left[\mathrm{Ca}^{2+}\right]_{i}$ in perfused hearts: critical evaluation of the 5F-BAPTA/NMR method as applied to the study of ischemia and reperfusion. Circ. Res. 66:1255-1267.

12. Kihara, Y., W. Grossman, and J. P. Morgan. 1989. Direct measurement of changes in intracellular calcium transients during hypoxia, ischemia, and reperfusion of the intact mammalian heart. Circ. Res. 65:1029-44.

13. Steenbergen, C., E. Murphy, L. Levy, and R. E. London. 1987. Elevation in cytosolic free calcium concentration early in myocardial ischemia in perfused rat heart. Circ. Res. 60:700-707.

14. Mohabir, R., H. K. Lee, R. W. Kurz, and W. T. Clusin. 1991. Effects of ischemia and hypercarbic acidosis on myocyte calcium transients, contraction, and $\mathrm{pH}_{\mathrm{i}}$ in perfused rabbit hearts. Circ. Res. 69:1525-1537.

15. Owen, P., S. Dennis, and L. H. Opie. 1990. Glucose flux rate regulates onset of ischemic contracture in globally underperfused rat hearts. Circ. Res. 66:344-354.

16. Kingsley, P. B., E. Y. Sako, M. Q. Yang, S. D. Zimmer, K. Ugurbil, J. E. Foker, and A. H. L. From. 1991. Ischemic contracture begins when anaerobic glycolysis stops: a ${ }^{31} \mathrm{P}-\mathrm{NMR}$ study of isolated rat hearts. Am. J. Physiol. 261:H469-H478.

17. Myears, D. W., B. E. Sobel, and S. Bergmann. 1987. Substrate use in ischemic and reperfused canine myocardium: quantitative considerations. $\mathrm{Am}$. J. Physiol. 253:H107-H114.

18. Lopaschuk, G. D., M. A. Spafford, N. J. Davies, and S. R. Wall. 1990. Glucose and palmitate oxidation in isolated working rat hearts reperfused after period of transient global ischemia. Circ. Res. 66:546-553.
19. Eberli, F. R., E. O. Weinberg, W. N. Grice, G. L. Horowitz, and C. S Apstein. 1991. Protective effect of increased glycolytic substrate against systolic and diastolic dysfunction and increased coronary resistance from prolonged global underperfusion and reperfusion in isolated rabbit hearts perfused with erythrocyte suspensions. Circ. Res. 68:466-481.

20. Jeremy, R. W., Y. Koretsune, E. Marban, and L. C. Becker. 1992. Relation between glycolysis and calcium homeostasis in postischemic myocardium. Circ. Res. 70:1180-1190.

21. Ikenouchi, H., O. Kohmoto, M. McMillan, and W. H. Barry. 1991. Contributions of $\left[\mathrm{Ca}^{2+}\right]_{i},\left[\mathrm{P}_{\mathrm{i}}\right]_{\mathrm{i}}$, and $\mathrm{pH}_{\mathrm{i}}$ to altered diastolic myocyte tone during partial metabolic inhibition. J. Clin. Invest. 88:55-61.

22. Corretti, M. C., Y. Koretsune, H. Kusuoka, V. P. Chacko, J. L. Zweier, and E. Marban. 1991. Glycolytic inhibition and calcium overload as consequences of exogenously generated free radicals in rabbit hearts. J. Clin. Invest. 88:1014-1025.

23. Nakamura, K., H. Kusuoka, G. Ambrosio, and L. C. Becker. 1993. Glycolysis is necessary to preserve myocardial $\mathrm{Ca}^{2+}$ homeostasis during $\beta$-adrenergic stimulation. Am. J. Physiol. 264:H670-H678.

24. Kusuoka, H., and E. Marban. 1992. Mechanism of the diastolic dysfunction induced by glycolytic inhibition: does ATP derived from glycolysis play a favored role in cellular $\mathrm{Ca}^{2+}$ homeostasis? Circulation. 86:I-748. (Abstr.)

25. Kusuoka, H., M. L. Weisfeldt, J. L. Zweier, W. E. Jacobus, and E. Marban. 1986. Mechanism of early contractile failure during hypoxia in intact ferret heart: evidence for modulation of maximal $\mathrm{Ca}^{2+}$-activated force by inorganic phosphate. Circ. Res. 59:270-282.

26. Allen, D. G., P. G. Morris, C. H. Orchard, and J. S. Pirolo. 1985. A nuclear magnetic resonance study of metabolism in the ferret heart during hypoxia and inhibition of glycolysis. J. Physiol. (Lond.). 361:185-204.

27. Marban, E., M. Kitakaze, H. Kusuoka, J. K. Porterfield, D. T. Yue, and V. P. Chacko. 1987. Intracellular free calcium concentration measured with ${ }^{19} \mathrm{~F}$ NMR spectroscopy in intact ferret hearts. Proc. Natl. Acad. Sci. USA. 842:60056009.

28. Marban, E., M. Kitakaze, V. P. Chacko, and M. M. Pike. 1988. Ca ${ }^{2+}$ transients in perfused hearts revealed by gated ${ }^{19} \mathrm{~F}$ NMR spectroscopy. Circ. Res. 63:673-678.

29. Smith, G. A., R. T. Hesketh, J. C. Metcalfe, J. Feeney, and P. G. Morris 1983. Intracellular calcium measurements by ${ }^{19} \mathrm{~F}$ NMR of fluorine-labelled chelators. Proc. Natl. Acad. Sci. USA. 80:7178-7182.

30. Vander Heide, R. S., K. A. Reimer, R. B. Jennings, and C. Steenbergen. 1992. Reduction of the osmotic load does not protect against ischemia-reperfusion injury. J. Mol. Cell. Cardiol. 24 (Suppl. III):S.15 (Abstr.)

31. Webb, J. L. 1966. Iodoacetate and iodoacetamide. In Enzyme and Metabolic Inhibitors. Volume 3. J. L. Webb, editor. Academic Press, Inc. New York. $1-283$.

32. Chatham, J., H. F. Gilbert, and G. K. Radda. 1988. Inhibition of glucose phosphorylation by fatty acids in the perfused rat heart. FEBS (Fed. Eur. Biochem. Soc.) Lett. 238:445-449.

33. Pirolo, J. S., and D. G. Allen. 1986. Assessment of techniques for preventing glycolysis in cardiac muscle. Cardiovasc. Res. 20:837-844.

34. Snedecor, G. W., and W. G. Cochran. 1989. Statistical Methods. 8th edition. Iowa State University Press, Ames, IA. 503pp.

35. Marban, E., and H. Kusuoka. 1987. Maximal Ca ${ }^{2+}$-activated force and myofilament $\mathrm{Ca}^{2+}$ sensitivity in intact mammalian hearts: differential effects of inorganic phosphate and hydrogen ions. J. Gen. Physiol. 90:609-623.

36. Lederer, W. J., E. Niggli, and R. W. Hadley. 1990. Sodium-calcium exchange in excitable cells: fuzzy space. Science (Wash. DC). 248:283.

37. Allen, D. G., and C. H. Orchard. 1987. Myocardial contractile function during ischemia and hypoxia. Circ. Res. 60:153-168.

38. Wyns, W., M. Schwaiger, S. C. Hung, D. B. Buxton, H. Hansen, C. Selin, R. Keen, M. E. Phelps, and H. R. Schelbert. 1989. Effects of inhibition of fatty acid oxidation on myocardial kinetics of "C-labeled palmitate. Circ. Res. 65:1787-1797.

39. Doorey, A. J., and W. H. Barry. 1983. The effects of inhibition of oxidative phosphorylation and glycolysis on contractility and high-energy phosphate content in cultured chick heart cells. Circ. Res. 53:192-201.

40. Barry, W. H., J. Pober, J. D. Marsh, S. R. Frankel, and T. W. Smith. 1980. Effects of graded hypoxia on contraction of cultured chick embryo ventricular cells. Am. J. Physiol. 239:H651-H567.

41. Bricknell, O. L., and L. H. Opie. 1978. Effects of substrates on tissue metabolic changes in the isolated rat heart during underperfusion and on release of lactate dehydrogenase and arrhythmias during reperfusion. Circ. Res. 43:102115 .

42. Higgins, T. J. C., D. Allsopp, P. J. Bailey, and E. D. A. D'Souza. 1981. The relationship between glycolysis, fatty acid metabolism and membrane integrity in neonatal myocytes. J. Mol. Cell. Cardiol. 13:599-615.

43. Paul, R. J., M. Bauer, and W. Pease. 1979. Vascular smooth muscle: Aerobic glycolysis linked to sodium and potassium transport processes. Science (Wash. DC). 206:1414-1416.

44. Auffermann W., S. Wagner, S. Wu, P. Buser, W. W. Parmley, and J. Wikman-Coffelt. 1990. Calcium inhibition of glycolysis contributes to ischemic injury. Cardiovasc. Res. 24:510-520. 